\title{
Some new mesoscopic crossover length scales concerning the Hamaker constant
}

\author{
ZHAO YaPu ${ }^{1,2 *}$ \\ ${ }^{1}$ State Key Laboratory of Nonlinear Mechanics (LNM), Institute of Mechanics, Chinese Academy of Sciences, Beijing 100190, China; \\ ${ }^{2}$ School of Engineering Science, University of Chinese Academy of Sciences, Beijing 100049, China
}

Received October 30, 2019; accepted November 20, 2019; published online November 27, 2019

Citation: Zhao Y P. Some new mesoscopic crossover length scales concerning the Hamaker constant. Sci China Tech Sci, 2019, 62: 2310-2312, https://doi.org/ 10.1007/s11431-019-1487-5

The last three decades have witnessed a global surge in nanoand micro-mechanics driven by the rapid development of nanotechnology, microelectromechanical systems (MEMS) and nanoelectromechanical systems (NEMS) [1]. Although a generally accepted framework is still lacking for mesoscopic mechanics, four broad categories of viewpoints have been agreed upon [2]:

(1) Continuum mechanics works quite well even for a few atoms in diameter. In 1997, Richard E. Smalley (1943 -2005), the late Nobel laureate, and Yakobson [3] once commented: "The behavior of the hollow tubules is complex, but still predictable with continuum-elastic methods. In its applications to nanotubes, the correspondence between the elastic model and molecular dynamics is remarkable. The laws of continuum mechanics are amazingly robust and allow one to treat even intrinsically discrete objects only a few atoms in diameter."

(2) The classical continuum mechanics is modifiable by surface or interface terms due to huge surface-to-volume ratios [4]. It keeps working when the surface or interface terms such as van der Waals (vdW) and Casimir interactions are introduced [5-11].

(3) The quasi-continuum method is based on the bottomup method [12,13].

(4) The Nobel Prize in Chemistry 2013 was awarded jointly to Martin Karplus, Michael Levitt and Arieh Warshel "for the development of multiscale models for complex chemical

*Corresponding author (email: yzhao@imech.ac.cn) systems". The QM/MM hybrid method has been widely used as a typical multiscale in mechanical systems $[14,15]$.

Kallmann and Willstaetter [16] ascribed the mutual attraction between particles to the vdW-London forces in 1932 . They made use of the additivity of the atomic interaction energies, which were proposed shortly before by Fritz Wolfgang London (1900-1954), to calculate the attraction between particles, but only for very large separations (for the purpose of the point approximation) while disregarding the medium. Bradley published a calculation for spheres and arbitrary power laws of attraction in 1932 [17], which used symbol $A$ for the material constant but contained some mistakes. Sparnaay [18] claimed that Sir Isaac Newton (1643-1727) had already made similar calculations.

In 1936, Jan Hendrik de Boer (1899-1971) considered the role of vdW forces in material cohesion and correctly calculated the interaction of flat slabs [19]. Just one year later, Hugo Christiaan Hamaker (1905-1993) published a heavily cited paper in Physica [20] on the double three-dimensional integration required in evaluating the London dispersive interaction between two spheres of arbitrary radii and distances, including the planes when the sphere radius approaches infinity and a general discussion of the role of the medium. The interaction energy obtained by the above integration is highly condensed into an expression consisting of two factors, i.e., a geometric factor depending on the size, shape, and separation of the bodies involved, and a material factor depending only on the material properties - the "constant" $A$. Thus the Hamaker constant with a dimension 
of energy was born, and this constant $A$ of the order of (0.4$4) \times 10^{-19} \mathrm{~J}$ can serve as the landmark energy to cross from continuum mechanics to mesoscopic mechanics.

Nowadays, instead of summing the effects of atoms, the absorption spectra are used to directly calculate the interaction between electromagnetic fluctuations in the bodies. This leads to a more reliable value of constant $A$; nevertheless, in the first-order approximation, the geometric factor remains unchanged.

The importance of Hamaker's formulation is evident in the modern designation of $A$ as the "Hamaker constant" as approved by the International Union of Pure and Applied Chemistry (IUPAC) [21] on July 23, 1971.

In 2019, we utilized the bottom-up method to study the peeling processes of a heterostructure bonded by the vdW force, the graphene/ $\mathrm{MoS}_{2}$ bilayer structure as illustrated in Figure 1. Firstly, some fundamental parameters such as lattice mismatch, strain energy densities and potential between graphene and $\mathrm{MoS}_{2}$ were obtained from density functional theory calculations with dispersion corrections (DFT-D2) [22]. Secondly, the peeling process was investigated subjected to various kinds of loadings by using molecular dynamics simulations. The changes in the total energy, elastic energy, vdW interaction potential and peeling force were obtained, and then the peeling forces obtained from these simulation results were compared with those from classical continuum methods. Thirdly, a mechanistic analysis of the peeling process was conducted and a quasi-continuum method was developed to describe the evolution of the peeling force. Finally, a new characteristic length, a crucial parameter that reflects the bending and interfacial properties of layered materials during peeling, was suggested by using the Hamaker constant. This study may assist in understanding the atomic-level mechanisms during the peeling of layered materials, and such mechanisms may provide some new insights into the automatic mechanical assembly of the $\mathrm{vdW}$ heterostructures by robots $[23,24]$.

Table 1 shows how the vdW interactions compete with

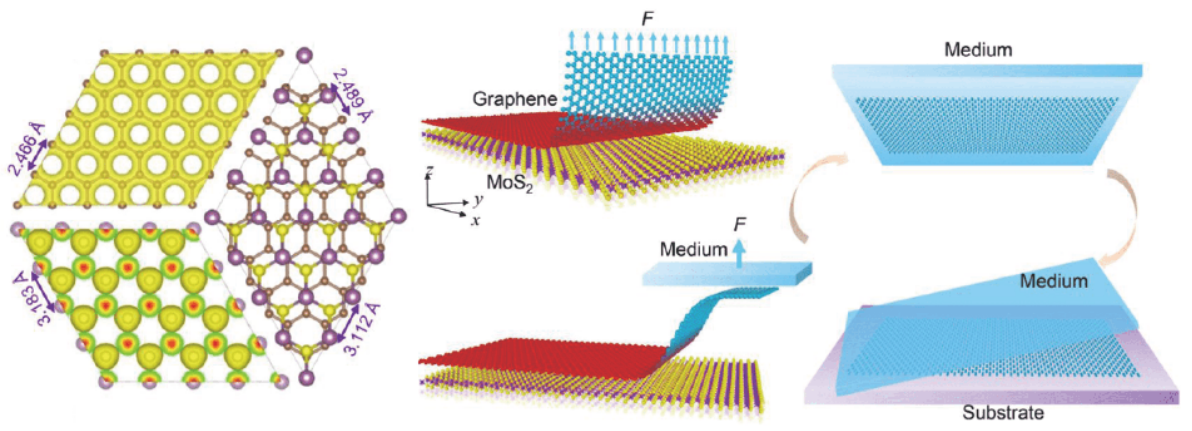

Figure 1 (Color online) Mechanical peeling of graphene/ $\mathrm{MoS}_{2} \mathrm{vdW}$ heterostructures [22].

Table 1 Some characteristic length scales related to the Hamaker constant $A$

\begin{tabular}{|c|c|c|}
\hline Length scale & Formula & Physical interpretation \\
\hline $\begin{array}{l}\text { Thickness of precursor film } \\
\text { (by de Gennes) }\end{array}$ & $a_{p}=\sqrt{\frac{A}{6 \pi \gamma_{l}}}$ & $\begin{array}{l}\text { Competition between the vdW interaction and capillarity. Typical thickness of precursor } \\
\text { film is of the order several } \AA . \gamma_{l v}-\text { liquid-vapor surface tension }\end{array}$ \\
\hline $\begin{array}{l}\text { Length of precursor film } \\
\quad \text { (by de Gennes) }\end{array}$ & $L_{p}=\frac{1}{C a} \sqrt{\frac{S A}{6 \pi \gamma_{l, 2}^{2}}}$ & $\begin{array}{l}\text { Competitions among spreading inertia, spreading parameter and vdW interactions. } \\
\qquad S^{-} \text {spreading parameter, } \mathrm{Ca}^{-} \text {capillary number }\end{array}$ \\
\hline Mesh size & $\xi \sim \sqrt[3]{\frac{A}{E}} \sim \sqrt[3]{\frac{A}{G}}$ & Competition between the vdW and elastic deformation energies \\
\hline $\begin{array}{l}\text { Detachment length of cantilever } \\
\text { (by the author) [25] }\end{array}$ & $L_{\text {detach }}=\mathrm{C} \times g \sqrt[4]{\frac{E t^{3}}{A}}$ & $\begin{array}{l}\text { Bending of cantilever under the action of the } \mathrm{vdW} \text { force. Adhesion/stiction occurs when } \\
\text { the cantilever length is longer than this length }\end{array}$ \\
\hline $\begin{array}{l}\text { Peeling length } \\
\text { (by the author) [22] }\end{array}$ & $L_{\text {peeling }}=4 \sigma_{0} \sqrt{\frac{2 \pi D}{A}}$ & $D=\frac{E h^{3}}{12\left(1-v^{2}\right)}-$ bending rigidity of plate, $\sigma_{0}-$ atom equilibrium distance \\
\hline $\begin{array}{l}\text { Bending length } \\
\text { (by the author) }\end{array}$ & $l_{\text {bending }}=\frac{E I}{A}$ & $\begin{array}{l}\text { Beam bending under the action of the vdW force. } E I-\text { bending rigidity of beam. Some } \\
\text { typical scales for the single-walled carbon nanotubes are presented in Table } 2\end{array}$ \\
\hline $\begin{array}{l}\text { Electrostatic length } \\
\text { (by the author) }\end{array}$ & $l_{\text {electric }}=\frac{e^{2}}{4 \pi \varepsilon_{r} \varepsilon_{0} A}$ & Competition between electrostatic and vdW interactions \\
\hline $\begin{array}{l}\text { Impact length } \\
\text { (by the author) }\end{array}$ & $l_{\text {impact }}=\sqrt[3]{\frac{A}{6 \pi \rho V^{2}}}$ & Competition between the vdW interactions and impact inertia $\rho V^{2}$ \\
\hline
\end{tabular}


Table 2 Length scales for single-walled carbon nanotube with different diameter $D$

\begin{tabular}{ccc}
\hline$D(\mathrm{~nm})$ & $l_{\min }(\mathrm{nm})$ & $l_{\max }(\mathrm{nm})$ \\
\hline 1 & 0.293 & 2.93 \\
2 & 2.63 & 26.3 \\
3 & 9.21 & 92.1 \\
4 & 22.26 & 222.6 \\
5 & 43.97 & 439.7 \\
6 & 76.55 & 765.5 \\
\hline
\end{tabular}

capillarity, elastic deformation, electrostatic interaction and impact inertia. Some characteristic scales are summarized and some new length scales are suggested by the author for the first time. These length scales will play vital roles when they are comparable with the characteristic length of the system in study.

This work was supported in part by the National Natural Science Foundation of China (Grant No. 51861145314, 11872363), the Chinese Academy of Sciences (CAS) through the CAS Key Research Program of Frontier Sciences (Grant No. QYZDJ-SSW-JSC019), and the CAS Strategic Priority Research Program (Grant No. XDB22040401).

1 Xu Z P, Zheng Q S. Micro- and nano-mechanics in China: A brief review of recent progress and perspectives. Sci China-Phys Mech Astron, 2018, 61: 074601

2 Zhao Y P. Nano and Mesoscopic Mechanics. Beijing: Science Press, 2014

3 Yakobson B I, Smalley R E. Fullerene nanotubes: $\mathrm{C}_{1,000,000}$ and beyond. Am Scientist, 1997, 85: 324-337

4 Zhao Y P. Physical Mechanics of Surfaces and Interfaces. Beijing: Science Press, 2012

5 Gao M N, Huang X F, Zhao Y P. Formation of wavy-ring crack in drying droplet of protein solutions. Sci China Tech Sci, 2018, 61: 949958

6 Wang Z L, Chen E H, Zhao Y P. The effect of surface anisotropy on contact angles and the characterization of elliptical cap droplets. Sci China Tech Sci, 2018, 61: 309-316

7 Yu Y S, Xia X L, Zheng X, et al. Quasi-static motion of microparticles at the depinning contact line of an evaporating droplet on PDMS surface. Sci China-Phys Mech Astron, 2017, 60: 094612
8 Wang X H, Shen W H, Huang X F, et al. Estimating the thickness of diffusive solid electrolyte interface. Sci China-Phys Mech Astron, 2017, 60: 064612

9 Yang F Q. Relation between surface stress and surface energy for an elastic sphere: Effects of deformation and Maxwell stress. Sci ChinaPhys Mech Astron, 2017, 60: 104621

10 Zhao Y P, Wang L S, Yu T X. Mechanics of adhesion in MEMS-A review. J Adhes Sci Technol, 2003, 17: 519-546

11 Zhu H, Zhao Y, He Z, et al. An elastic-plastic contact model for line contact structures. Sci China-Phys Mech Astron, 2018, 61: 054611

12 Tadmor E B, Ortiz M, Phillips R. Quasicontinuum analysis of defects in solids. Philos Mag A, 1996, 73: 1529-1563

13 Shenoy V B, Miller R, Tadmor E B, et al. Quasicontinuum models of interfacial structure and deformation. Phys Rev Lett, 1998, 80: 742745

14 Yang Z, Zhao Y P. QM/MM and classical molecular dynamics simulation of histidine-tagged peptide immobilization on nickel surface. Mater Sci Eng-A, 2006, 423: 84-91

15 Yin J, Zhao Y P. Hybrid QM/MM simulation of the hydration phenomena of dipalmitoylphosphatidylcholine headgroup. J Colloid Interface Sci, 2009, 329: 410-415

16 Kallmann H, Willstaetter M. Zur theorie des aufbaues kolloidaler systeme. Naturwissenschaften, 1932, 20: 952-953

17 Bradley R S. LXXIX. The cohesive force between solid surfaces and the surface energy of solids. London Edinburgh Dublin Philos Mag J Sci, 1932, 13: 853-862

18 Sparnaay M J. Four notes on van der Waals forces. Induction effect, nonadditivity, attraction between a cone and a flat plate (asperities), history. J Colloid Interface Sci, 1983, 91: 307-319

19 de Boer J H. The influence of van der Waals' forces and primary bonds on binding energy, strength and orientation, with special reference to some artificial resins. Trans Faraday Soc, 1936, 32: 10-37

20 Hamaker H C. The London-van der Waals attraction between spherical particles. Physica, 1937, 4: 1058-1072

21 Mysels K J, Scholten P C. C\&SC Folklore. 4. H. C. Hamaker, more than a constant. Langmuir, 1991, 7: 209-211

22 Lin K, Zhao Y P. Mechanical peeling of van der Waals heterostructures: Theory and simulations. Extreme Mech Lett, 2019, 30: 100501

23 Masubuchi S, Morimoto M, Morikawa S, et al. Autonomous robotic searching and assembly of two-dimensional crystals to build van der Waals superlattices. Nat Commun, 2018, 9: 1413

24 Wang Y, Kim J C, Wu R J, et al. Van der Waals contacts between three-dimensional metals and two-dimensional semiconductors. Nature, 2019, 568: 70-74

25 Lin W H, Zhao Y P. Dynamic behaviour of nanoscale electrostatic actuators. Chin Phys Lett, 2003, 20: 2070-2073 